\title{
Micro-Pulse, Differential Absorption Lidar (DIAL) Network FOR MEASURing THE SPATIAL AND TEMPORAL DisTRIBUTION OF WATER VAPOR IN THE LOWER ATMOSPHERE
}

\author{
Scott Spuler ${ }^{* 1}$, Kevin Repasky ${ }^{2}$, Matt Hayman ${ }^{1}$, Amin Nehrir ${ }^{3}$ \\ ${ }^{1}$ National Center for Atmospheric Research, Earth Observing Lab, Boulder, CO 80307, USA, * spuler@ucar.edu \\ ${ }^{2}$ Montana State University, Electrical and Computer Engineering, Bozeman, MT 59717, USA \\ ${ }^{3}$ NASA Langley Research Center, Hampton, VA 23681, USA
}

\begin{abstract}
The National Center for Atmospheric Research (NCAR) and Montana State Univeristy (MSU) are developing a test network of five micro-pulse differential absorption lidars to continuously measure high-vertical-resolution water vapor in the lower atmosphere. The instruments are accurate, yet low-cost; operate unattended, and eye-safe - all key features to enable the larger network needed to characterize atmospheric moisture variability which influences important processes related to weather and climate.
\end{abstract}

\section{INTRODUCTION}

Water vapor is one of the fundamental thermodynamic variables that define the state of the atmosphere. It is highly variable in space and time and influences many important processes related to weather and climate. Three recent National Research Council (NRC) reports highlight the need for improved water vapor measurements as a necessary step toward improving mesoscale numerical weather prediction and quantitative precipitation forecasting skills $[1,2,3]$. A recent review paper detailing the state of the art for thermodynamic profiling, [4] also stresses the importance of water vapor profiling in the lower troposphere and suggests low-power water vapor differential absorption lidar (DIAL) as a promising candidate for a compact remote sensing instrument that can be deployed in networks.

Developments in semiconductor laser and amplifier technology have enabled low cost, compact lasers with narrow linewidths and long lifetimes. Semiconductor lasers are well suited to lidar systems designed for network deployment where the instrument needs to be capable of unattended operation for long periods without maintenance and should not pose a risk to aircraft or bystanders. Technology using a narrow-line semiconductor laser, such as external cavity laser diodes (ECDL), to seed a tapered semiconductor optical amplifier (TSOA) were developed and demonstrated for the purpose of water vapor profiling using the differential absorption lidar technique at $828 \mathrm{~nm}[5,6,7]$. Following this seminal work, a field-capable micro-pulse DIAL (MPD) instrument was collaboratively designed, built and tested by MSU and NCAR $[8,9]$. This eye-safe and reliable design has been shown to deliver accurate retrievals of water vapor in the lower troposphere and produce scientifically significant data [10].
NCAR and MSU are now developing a five unit water vapor profiling testbed - necessary to capture nonlinear mesoscale moisture variability caused by land surface effects, such as terrain variations, lakes, vegetation and irrigation. Since water vapor is highly variable in time and three-dimensional space, five stations allow for measuring mean water vapor values within four triangular areas and could quantify non-linearities in gradients across the network domain. The addition of wind profile measurements collocated with each of these stations would provide for information on moisture advection and moisture divergence.

\section{INSTRUMENT DESIGN}

The instrument, shown schematically in Figure 1, utilizes a diode-laser-based master oscillator power amplifier (MOPA) configured transmitter, and a shared telescope transmitter and receiver to achieve opto-mechanically stable, and eye-safe, operation. A multistage filtered receiver is implemented to allow continuous measurement in high background conditions (e.g., bright daytime clouds).

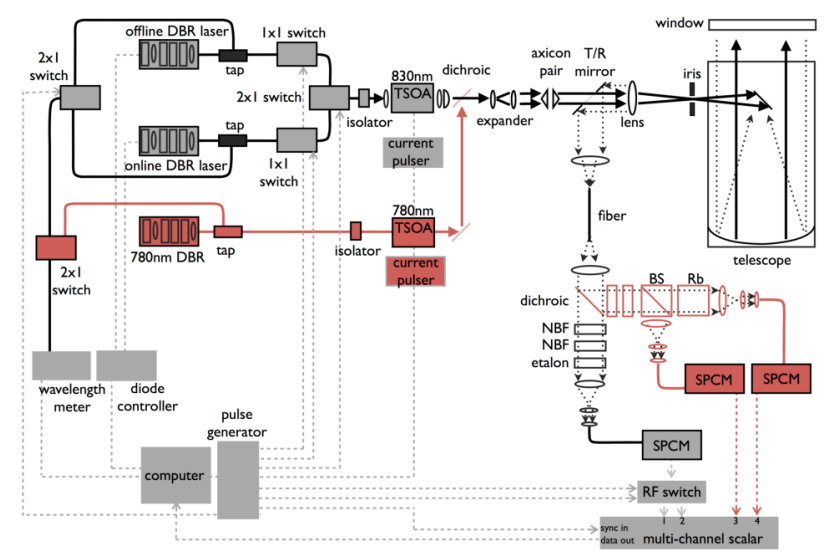

Figure 1. Schematic of the current MPD system combined with a new channel (shown in red) - a diode-laser-based (DLB) High Spectral Resolution Lidar (HSRL). $\mathrm{BS}=$ beam-splitter; $\mathrm{T} / \mathrm{R}=$ transmit $\mathrm{re}$ ceive; $\mathrm{Rb}=$ Rubidium vapor cell; $\mathrm{SPCM}=$ Single Photon Counting Module.

A pair of distributed Bragg reflector (DBR) semiconduc- 
tor lasers nominally centered at $828 \mathrm{~nm}$ are interleaved at $100 \mathrm{~Hz}$ and used to seed a TSOA. Current pulses to the TOSA amplify the laser and produce approximately $5 \mu \mathrm{J}$ over 900 ns duration. The beam is shaped into an annular ring with an axicon pair and then expanded to fill the inner part of the telescope with a radius of $10 \mathrm{~cm}$. The laser repetition rate, set to $7 \mathrm{kHz}$, limits the maximum altitude of the lidar system to $21 \mathrm{~km}$. Direct exposure to the laser exiting the telescope is within eye-safe limits. The backscattered light received is directed into a $105 \mu \mathrm{m}$ core multimode fiber in the receiver system. The light from the fiber is collimated and passes through a pair of interference filters and solid etalon, with a combined passband of $0.014 \mathrm{~nm}$ for daylight rejection. The filtered light is then directed into a fiber coupled single photon counting module (SCPM), which utilizes a silicon avalanche photodiode operated in so-called 'Geiger-mode'.

To date, the NCAR water vapor MPD has been deployed at two field campaigns - FRAPPE (Colorado, USA) in the summer of 2014, and PECAN (Kansas, USA) in the summer of 2015 within a $6.1 \mathrm{~m}$ long intermodal shipping container equipped as mobile lidar laboratory. The system was run unattended in all weather and provided nearcontinuous profiles of water vapor - including periods of bright daytime clouds - from $300 \mathrm{~m}$ above ground level to $4 \mathrm{~km}$ (or cloud base, whichever comes first) with $150 \mathrm{~m}$ nominal vertical resolution and 1-5 minute temporal resolution. The MPD was greater than $95 \%$ operational in both field projects resulting in a high continuity of water vapor profile time series as shown in Fig. 2. Well-established water vapor observing systems, including radiosondes, infrared and microwave radiometers, and ground-based global positioning system receivers were collocated with the MPD at these field campaigns. Data comparisons showed excellent agreement between the MPD and these systems, with the MPD providing distinct operational and resolution advantages [10]. An example comparison with a series of radiosondes is shown as Fig. 3. The continuous water vapor measured by MPD agrees well with the 11 radiosonde launched during this $12 \mathrm{hr}$ time period. Notably, the MPD was the only one of three collocated continuously profiling instruments that detected the two elevated moist layers from 1 to $3 \mathrm{~km}$ AGL during this time period. [10]

Following the PECAN deployment, a more portable field enclosure was constructed to simplify fielding the water vapor MPD. A picture of the field prototype from Feb 2016 is shown as Fig. 4. The new enclosure was designed to 1.) be reasonably small and lightweight so that it could be moved with a forklift and not require a crane for lifting on/off transport vehicles, 2.) provide a weatherproof and thermally stable environment for the instrument, and 3.) provide instrument vibration isolation, to minimize the amount of disassembly and packing needed for shipment to field projects. The enclosure has external dimensions of $1 \mathrm{~m}$ depth, $2 \mathrm{~m}$ width, and $2 \mathrm{~m}$ height and was designed to have extra space around the instrument to allow easy alignment access and allow for future changes and upgrades.

NCAR has already demonstrated one potential upgrade technology - a diode-laser-based (DLB) high spectral resolution lidar (HSRL) channel based on the same lowcost lidar architecture [11, 12]. This HSRL channel, is added to the MPD as shown in Figure 1. The upgraded instrument combines water vapor with quantitative measurements of aerosols and could significantly contribute to studies of aerosol-cloud interaction. There is additional synergy as HSRL directly measures the aerosolto-molecular backscatter ratio and would provide accurate Rayleigh Doppler (RD) corrections for the water vapor spectroscopy (although this correction term is small, since the MPD instrument was designed to be insensitive to RD errors [10]). In addition, HSRL do not require a correction for the geometric overlap function to obtain the aerosol backscatter coefficient and are well suited to quantitative retrievals at comparatively low altitudes. As part of the network deployment, the DLB-HSRL offers opportunities for higher resolution measurements of mixing layer height for model and satellite comparison studies as well as continuous monitoring of a key observational parameter for weather and climate.

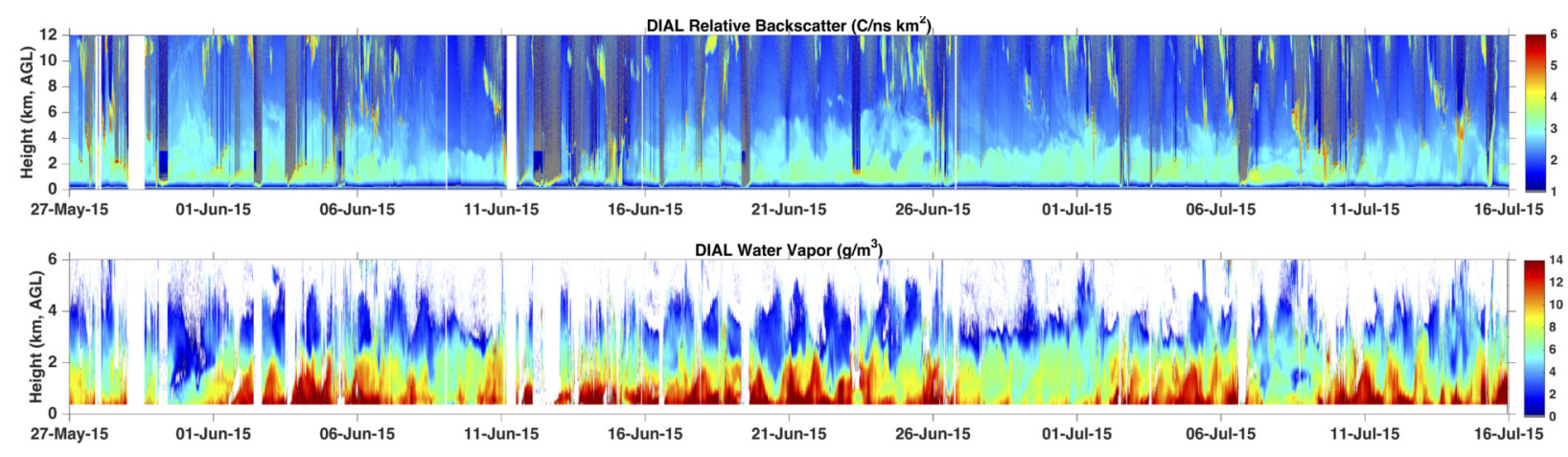

Figure 2. Time series of decimated 5-min/75-m MPD profiles showing (top) range-corrected relative backscatter and (bottom) absolute humidity $\left(\mathrm{gm}^{-3}\right)$. Vertical white bars signify missing data due to MPD downtime or attenuated signal above optically thick clouds. 


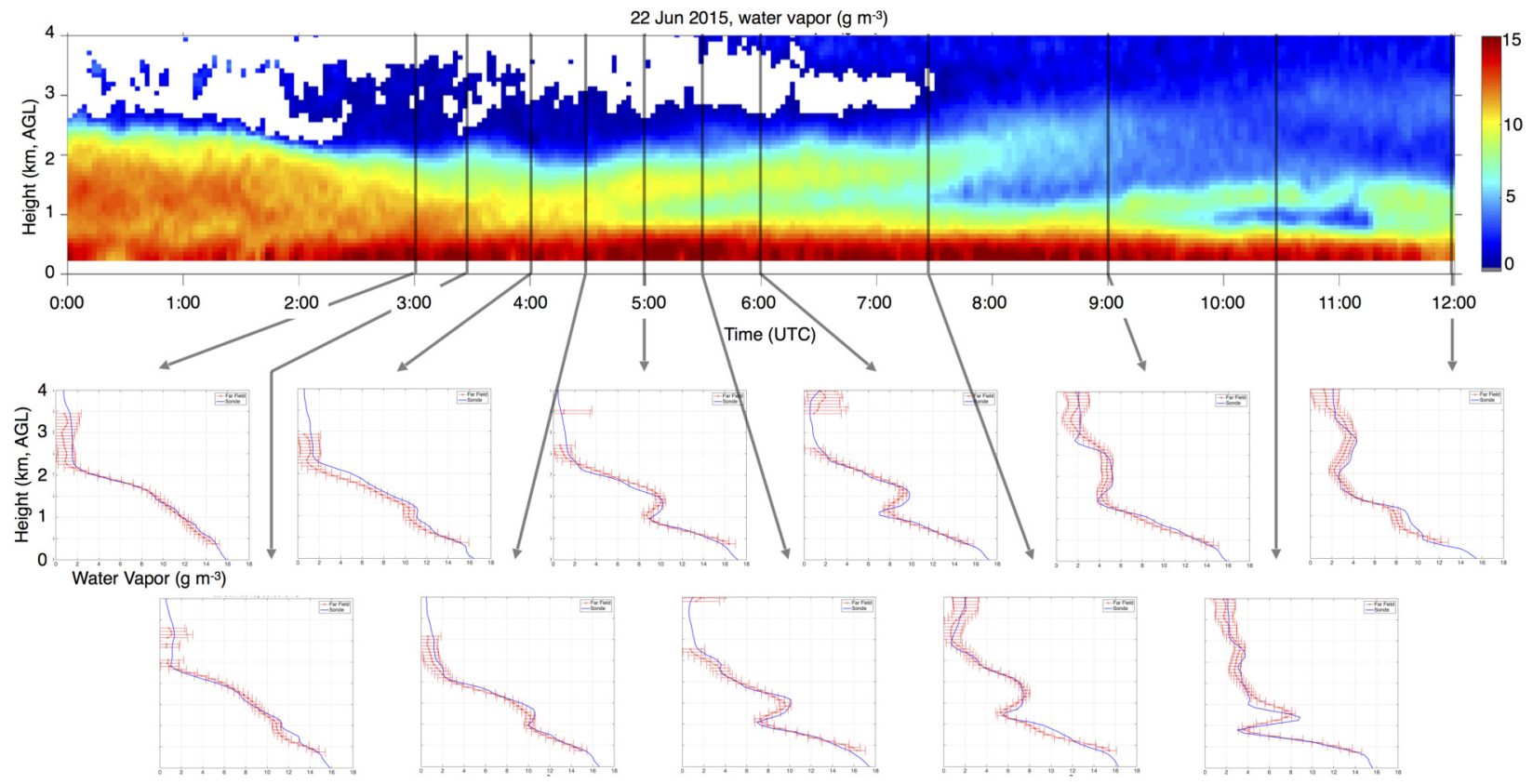

Figure 3. Water vapor profile time series measured with the MPD on 22-Jun-2015. The profiles show elevated layers of water vapor during the $12 \mathrm{hr}$ time span. The gray lines indicate sonde launch times and point to the corresponding comparison profiles. Water vapor profiles measured by the sonde and MPD are shown in blue and red; respectively. The horizontal red lines represent the MPD measurement error at each 75-m height interval.

MSU and NCAR have also developed a performance model for a low-cost temperature channel to integrate into the MPD architecture. For the water vapor DIAL, a temperature insensitive absorption feature was chosen, and the backscatter signal from the online and offline wavelengths are used to retrieve the number density profile as a function of range. However, if a temperature sensitive absorption feature is chosen for a molecule with a well know atmospheric mixing ratio such as oxygen $\left(\mathrm{O}_{2}\right)$, temperature as a function of range can be retrieved via the DIAL technique. For low error DIAL temperature retrievals (e.g., <1C), the aerosol-to-molecular backscatter ratio must be known [14]. This value can be obtained from the DLB-HSRL which is close in wavelength. The estimated performance of the proposed oxygen MPD temperature channel is comparable to a rotational Raman lidar [15].

\section{NETWORK CONSTRUCTION PLANS}

At the time of this writing, two fully functional water vapor MPD instruments exist (only one unit has been packaged into its field environmental enclosure). These two units are being modified to match one another and after the design is finalized, three cloned units will be built. All of the units will include design modifications to improve reliability in the field, e.g., one potential upgrade is a compact seed laser package with integrated optics to provide a fiber coupled and optically isolated output. This upgrade effort is part of a NASA funded SBIR [13] and would reduce alignment time at the start of new field deployments. The instrument environmental enclosure, based on the current design, will allow for long term deployments and include temperature control and remote internet access. Additionally, the instrument control will be modified to improve reliability for the

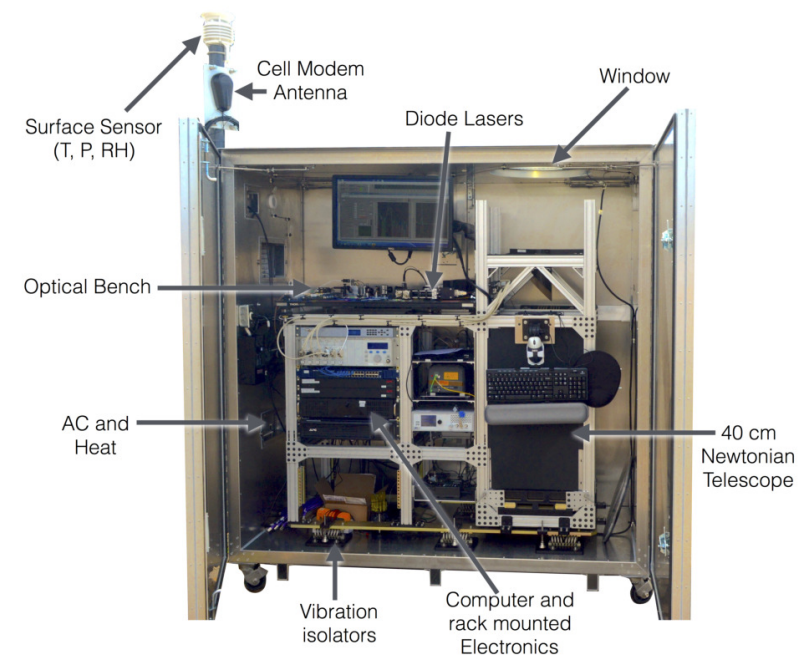

Figure 4. Picture of the MPD system within its portable field enclosure. The unit (with a size of $1 \mathrm{~m} \times 2 \mathrm{~m} \times 2 \mathrm{~m}$ ) is the first of the small network. The next units to be built will be similar in design although slightly smaller 
network by incorporating instrument monitoring, alarm, and control features. Processing routines have been automated and will provide the data in an appropriate format for ease of data assimilation. When the construction phase is complete, each MPD instrument will be tested using radiosondes for validation. The MPD testbed is anticipated to be ready for service in 2019, and will be made available to the larger research community for field campaigns and deployment through NCAR.

\section{SUMMARY}

A small testbed network of validated micro-pulse water vapor DIAL instruments is under construction. The diode-laser-based transmitter is inherently lowmaintenance, low-cost, and designed to be eye-safe at the exit port. Each node provides continuous profiles of water vapor with complete daytime coverage - including periods of bright clouds - from $300 \mathrm{~m}$ above ground level to $4 \mathrm{~km}$ (or cloud base, whichever comes first) with 150 $\mathrm{m}$ nominal vertical resolution and 1 to 5 minute temporal resolution. A low-cost HSRL channel can readily be integrated into the instrument which would significantly contribute to studies of aerosol-cloud interaction. The five unit testbed network has a planned completion date in 2019. The testbed will be a significant step towards improving our understanding of the distribution of atmospheric water vapor, and provide the opportunity to measure the impact potential of a national scale network.

\section{ACKNOWLEDGMENTS}

NCAR is sponsored by the National Science Foundation. The authors would like to acknowledge the support of the National Science Foundation grant number 1624736.

\section{REFERENCES}

[1] National Research Council, 2009: Observing Weather and Climate from the Ground Up: A Nationwide Network of Networks. The National Academies Press, Washington, DC.

[2] National Research Council, 2010: When Weather Matters: Science and Service to Meet Critical Societal Needs. The National Academies Press, Washington, DC.

[3] National Research Council, 2012: Weather Services for the Nation: Becoming Second to None. The National Academies Press, Washington, DC.

[4] Wulfmeyer, V., R. M. Hardesty, D. D. Turner, A. Behrendt, M. P. Cadeddu, P. Di Girolamo, P. Schlssel, J. Van Baelen, and F. Zus, 2015: A review of the remote sensing of lower tropospheric thermodynamic profiles and its indispensable role for the understanding and the simulation of water and energy cycles Rev. Geophys., 53, 819-895.

[5] Nehrir, A. R., Repasky, K. S., Carlsten, J. L., Obland, M. D., and Shaw, J. A., 2009: Water Vapor Profiling Using a Widely Tunable, Amplified Diode-Laser-Based Differential Absorption Lidar
(DIAL). Journal of Atmospheric and Oceanic Technology, 26(4), 733-745.

[6] Nehrir, A. R., Repasky, K. S., and Carlsten, J. L., 2011: Eye-Safe Diode-Laser-Based Micropulse Differential Absorption Lidar (DIAL) for Water Vapor Profiling in the Lower Troposphere. Journal of Atmospheric and Oceanic Technology, 28(2), 131147.

[7] Nehrir, A. R., Repasky, K. S., and Carlsten, J. L., 2012: Micropulse water vapor differential absorption lidar: transmitter design and performance. $O p$ tics express, 20(22), 25137-51.

[8] Repasky, K., Moen, D., Spuler, S., Nehrir, A., and Carlsten, J., 2013: Progress towards an Autonomous Field Deployable Diode- Laser-Based Differential Absorption Lidar (DIAL) for Profiling Water Vapor in the Lower Troposphere. Remote Sens., 5, 6241-6259.

[9] Spuler, S. M., Repasky, K. S., Morley, B., Moen, D., Hayman, M., and Nehrir, A. R., 2015: Field deployable diode-laser-based differential absorption lidar (DIAL) for profiling water vapor. Atmospheric Measurement Techniques, 8, 1073-1087.

[10] Weckwerth, T. M., K. Weber, D. D. Turner, and S. M. Spuler, 2016: Validation of a Water Vapor Micropulse Differential Absorption Lidar (DIAL). J. Atmospheric and Oceanic Technology, 33, 23532372.

[11] Hayman, M., S. Spuler, B. Morley, and E.W. Eloranta, 2015: Design of a low cost diode-laser-based high spectral resolution lidar. 27th International Laser Radar Conference, New York, NY, USA.

[12] Hayman, M., and S. Spuler, 2017: Demonstration of a low cost diode-laser-based high spectral resolution lidar (HSRL). 28th International Laser Radar Conference, Bucharest, Romania.

[13] Overton G, 2016: Lidar: Differential absorption lidar demands high-power, narrowband DBR sources Laser Focus World, 52(9), 14-15.

[14] Bösenberg, J., 1998: Ground-based differential absorption lidar for water-vapor and temperature profiling: methodology. Applied Optics, 37(18), 38453860.

[15] Repasky, K., Spuler, S., and Hayman, M., 2017: Modeling of a Semiconductor Based Differential Absorption Lidar for Temperature Profiling in the Lower Troposphere AMS Annual Meeting, Seattle WA, USA 24-Jan-2017 . 